\title{
Infected Superior Mesenteric Artery Aneurysm
}

\author{
Abigail Fong, $\mathrm{MD}^{1}$ Rakesh Navuluri, $\mathrm{MD}^{2}$ \\ ${ }^{1}$ The University of Chicago, Pritzker School of Medicine, Chicago, \\ Illinois \\ 2 Section of Vascular and Interventional Radiology, Department of \\ Radiology, University of Chicago, Chicago, Illinois
}

Semin Intervent Radiol 2016;33:61-64
Address for correspondence Rakesh Navuluri, MD, Section of Vascular and Interventional Radiology, Department of Radiology, University of Chicago Medicine, 5841 S. Maryland Avenue, MC 2026, Room Q-219, Chicago, IL 60637 (e-mail: RNavuluri@radiology.bsd.uchicago.edu).

\section{Case Report}

A 71-year-old woman presented to the emergency department with right upper quadrant abdominal pain and vomiting of 3 days. The patient was afebrile and white blood cell (WBC) count was within normal limits. The patient has a complex past medical history including left ventricular assist device (LVAD) that was complicated by methicillin-sensitive Staphylococcus aureus (MSSA) infection, and treated with 6 weeks of intravenous (IV) vancomycin. She was found to have methicillin-resistant $S$. aureus (MRSA)-positive blood cultures, and a computed tomographic (CT) scan revealed a $3.5 \times 3.0 \times 5.3 \mathrm{~cm}$ superior mesenteric artery (SMA) aneurysm (-Fig. 1). Additional findings included a $2.2 \times 2.3 \times 2.1 \mathrm{~cm}$ gastroepiploic aneurysm and multiple enhancing splenic lesions, suspicious for abscesses versus mycotic pseudoaneurysms.

Interventional radiologist recommended embolization after being consulted regarding treatment of the aneurysm. Following sedation and local anesthetic administration, the right common femoral artery was accessed and a $5 \mathrm{~F}$ vascular sheath (Terumo, Tokyo, Japan) was placed. Next a 5F pigtail catheter was used to perform a flush aortogram (not shown), which showed no aortic abnormalities. An RC1 catheter (Cook Medical, Bloomington, IN) was used to catheterize the celiac artery and perform celiac angiography which revealed the large pseudoaneurysm. A Renegade STC 18 microcatheter (Boston Scientific, Marlborough, MA) was advanced into the lumen of the SMA aneurysm (-Fig. 2a). Several microcoils (Interlock, Boston Scientific, Marlborough, MA) were placed into the aneurysm to promote thrombosis (-Fig. 2b). Because of the perceived risk of intraprocedural rupture, the aneurysm sac was not packed with additional coils. Instead, a 5.3-mm MVP microvascular plug (Covidien, Irvine, CA) was deployed in the proximal artery to seal the aneurysm neck (-Fig. 2c). Repeat angiogram of the SMA showed successful placement of the microvascular plug in the neck of the aneurysm and complete occlusion of the pseudoaneurysm. A second distal gastroepiploic pseudoaneurysm was then embolized with microcoils (not shown). A CT angiography CTA was performed the following day demonstrating successful embolization of both aneurysms (-Fig. 3).

\section{Discussion}

Visceral aneurysms are uncommon, comprising only 0.1 to $0.2 \%$ of aneurysms, though the true incidence may be higher due to asymptomatic occurrence ${ }^{1}$ (-Table 1). Splenic aneurysms are most common; SMA aneurysms are rare, accounting for only $5.5 \%$ of all visceral artery aneurysms. ${ }^{2}$ SMA aneurysms, in contrast to many other types of visceral aneurysms, are often symptomatic, causing pain, nausea, hemorrhage, or jaundice. ${ }^{1}$ Additionally, mural thrombus is often present and can lead to symptoms if embolization of the thrombus or narrowing of the vascular lumen occurs. ${ }^{3}$ SMA aneurysms have a reported risk of rupture of approximately $50 \%{ }^{3}$ Depending on the characteristics of the aneurysm, especially size and location, the mortality from visceral aneurysm rupture is high (25-100\%). ${ }^{4}$

Risk factors for infected aneurysms are those that increase the odds of infection occurrence or vascular inoculation. These factors include arterial injury, preceding infection, atherosclerosis, preexisting aneurysm, and immunocompromise. These risk factors can lead to the direct inoculation, bacterial seeding, or mycotic/embolic spread of infection to the site of the aneurysm.

With the advent and use of cross-sectional imaging, many asymptomatic aneurysms are now discovered. Most visceral aneurysms are readily diagnosed on CT, CTA, or
Issue Theme Dialysis Interventions; Guest Editor, Gordon McLennan, MD, FSIR
Copyright (c) 2016 by Thieme Medical Publishers, Inc., 333 Seventh Avenue, New York, NY 10001, USA.

Tel: +1(212) 584-4662.
DOI http://dx.doi.org/ 10.1055/s-0036-1572358. ISSN 0739-9529. 

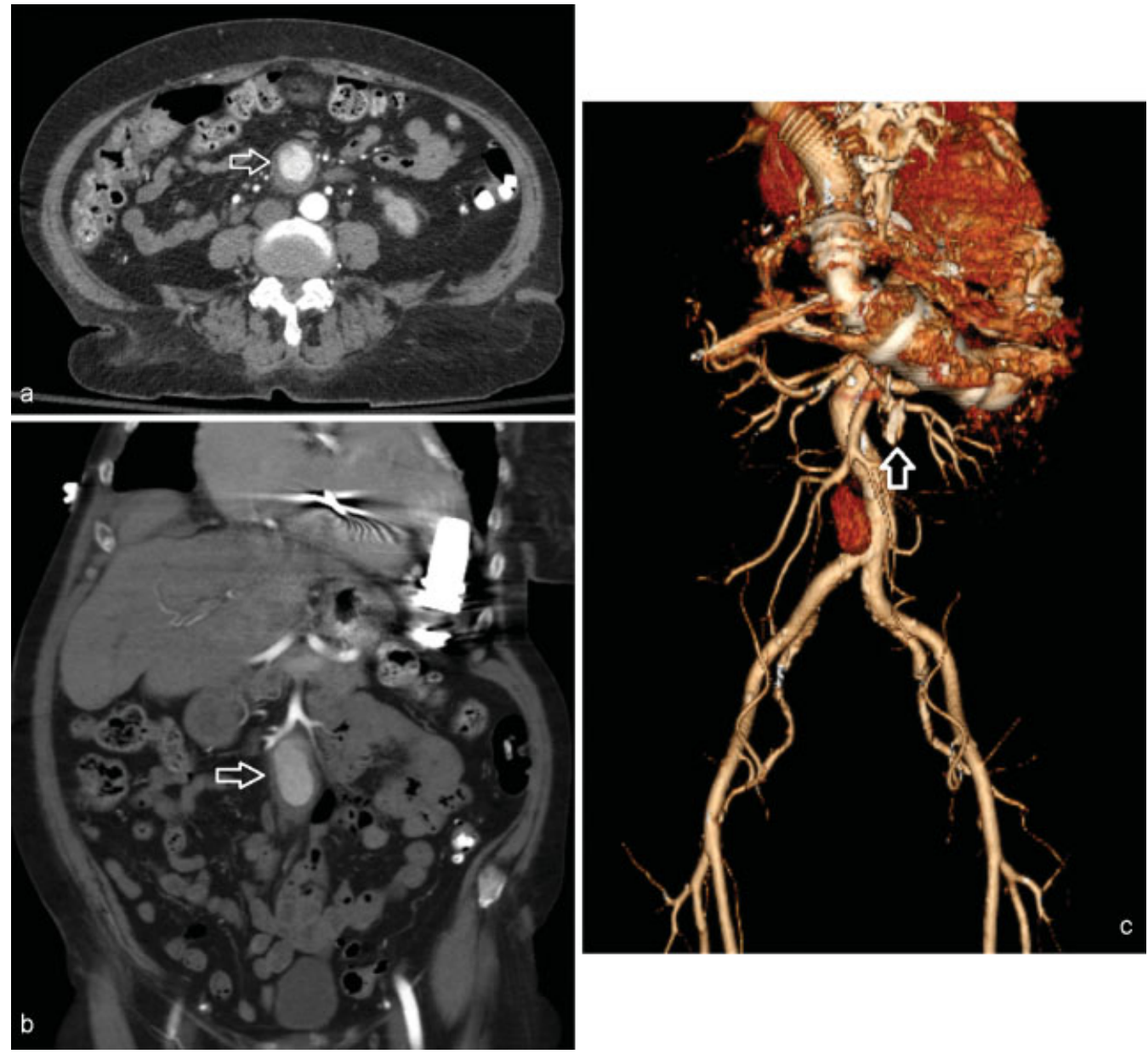

Fig. 1 (a-c) Enhanced axial, coronal, and 3D CTA images demonstrating a large SMA aneurysm (arrows).

magnetic resonance angiography. Ultrasound can also be used to identify the presence of aneurysms, although the sensitivity of ultrasound is likely lower due to overlying bowel. Imaging features that suggest possible infectious etiologies of aneurysms include saccular or eccentrically shaped aneurysm, intramural air or collection of air around the vessel, rapid aneurysm enlargement, perivascular inflammation or fluid collection.

The etiology of SMA aneurysms has historically been infection, as was hypothesized in this case. Of these, Streptococcal and Staphylococcal species are the most common agents isolated from blood and tissue cultures, but often no organism is identified. ${ }^{2}$ SMA aneurysm rupture rate is higher than for other visceral aneurysms at 38 to $50 \%$, and rupture has high overall mortality rate of approximately $30 \%{ }^{2}$ Additionally, infected aneurysms have the added risk of spread of infection in addition to rupture, and without treatment can progress to fulminant sepsis. Owing to these risks, it is generally advised to treat all known infected aneurysms. This treatment comprises administration of antibiotics, generally before intervention, followed by surgical or endovascular intervention.

Endovascular intervention has been demonstrated to be a viable methodology for treatment of infected aneurysms, and can comprise the placement of stents, coils, glue, endovascular plugs, or thrombin. ${ }^{5}$ One case series found a $98 \%$ technical success rate in endovascular treatment of visceral aneurysms of all etiologies. ${ }^{6}$ For small, unruptured aneurysms in stable patients, however, antibiotics alone can be trialed, followed by surveillance and intervention as needed. Surveillance should be performed at short intervals, as infected aneurysms can rapidly expand and rupture over the course of a few days. Endovascular techniques provide a less invasive approach to treatment compared with surgery, but do not remove the focus of infection and must be performed in conjunction with antibiotics. Stents or stent grafts can be used to exclude aneurysms, and have been successfully used in the aorta, cerebral arteries, and other vascular beds as well. These require adequate landing zones on either side of the aneurysm and retain the risk of persistent infection, which has been reported in $23 \%$ of cases in one study 

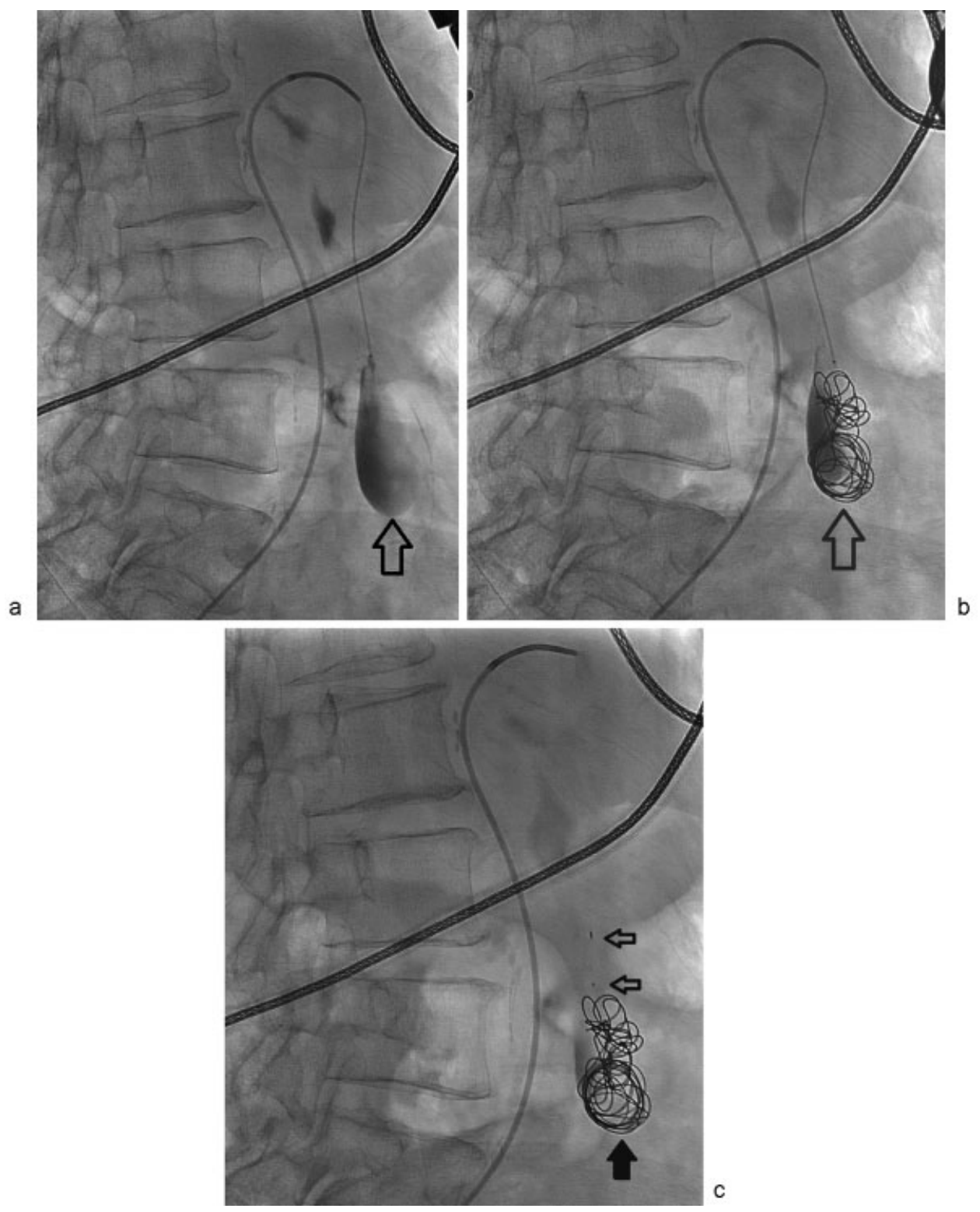

Fig. 2 (a) Spot fluoroscopic image demonstrating superior mesenteric artery (SMA) aneurysm during angiography (arrow). (b) Microcoil deployment within SMA aneurysm (arrow). (c) Intraprocedural image demonstrating successful coil (arrow) and vascular plug (open arrows) embolization of SMA aneurysm.

evaluating stented infected aortic aneurysms. ${ }^{7}$ Embolization, with coils, glue, or thrombin, is typically used as monotherapy in aneurysms with a narrow neck. Wide neck aneurysms may require hybrid techniques such as coil and stent/stent graft deployment, have a higher risk of therapy failure, and can be complicated by nontarget SMA occlusion and resulting bowel ischemia. Overall, endovascular approaches have been shown to have high technical success rates in occluding visceral aneurysms.

\section{Summary}

Infected aneurysms of the SMA are an uncommon condition. The data about the treatment of such aneurysms are limited by a lack of randomized trials and is governed by physician experience and case series. With increased use of endovascular techniques, however, the treatment of infected aneurysms is evolving and creating an increasing role for interventional radiology. 


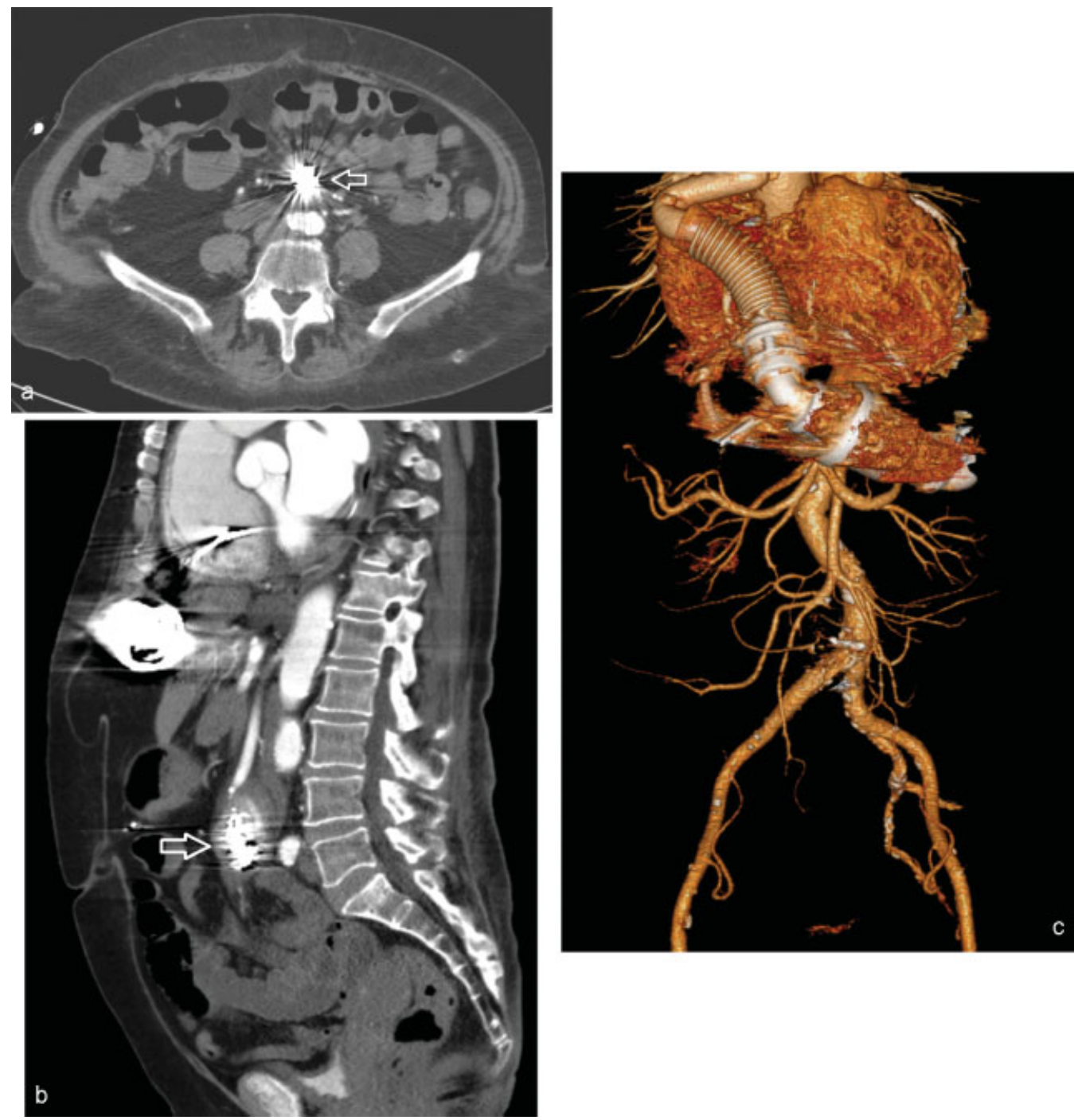

Fig. 3 (a-c) Enhanced axial, sagittal and 3D CTA images demonstrating successful embolization of superior mesenteric artery aneurysm (arrow).

Table 1 Most common visceral artery aneurysms

\begin{tabular}{|l|}
\hline 1. Splenic artery $(60-80 \%)$ \\
\hline 2. Hepatic artery (20\%) \\
\hline $\begin{array}{l}\text { 3. Gastroduodenal, pancreaticoduodenal, or pancreatic } \\
\text { artery (6\%) }\end{array}$ \\
\hline 4. Superior mesenteric artery $(5.5 \%)$ \\
\hline 5. Celiac artery (4\%) \\
\hline 6. Inferior mesenteric artery (1\%) \\
\hline
\end{tabular}

\section{References}

1 Drescher R, Köster O, von Rothenburg T. Superior mesenteric artery aneurysm stent graft. Abdom Imaging 2006;31(1):113-116
2 Sharma G, Semel ME, McGillicuddy EA, Ho KJ, Menard MT, Gates JD. Ruptured and unruptured mycotic superior mesenteric artery aneurysms. Ann Vasc Surg 2014;28(8): 1931.e5-1931.e8

3 Chadha M, Ahuja C. Visceral artery aneurysms: diagnosis and percutaneous management. Semin Intervent Radiol 2009;26(3): 196-206

4 Hemp JH, Sabri SS. Endovascular management of visceral arterial aneurysms. Tech Vasc Interv Radiol 2015;18(1):14-23

5 Lee WK, Mossop PJ, Little AF, et al. Infected (mycotic) aneurysms: spectrum of imaging appearances and management. Radiographics 2008;28(7):1853-1868

6 Tulsyan N, Kashyap VS, Greenberg RK, et al. The endovascular management of visceral artery aneurysms and pseudoaneurysms. J Vasc Surg 2007;45(2):276-283, discussion 283

7 Kan CD, Lee HL, Yang YJ. Outcome after endovascular stent graft treatment for mycotic aortic aneurysm: a systematic review. J Vasc Surg 2007;46(5):906-912 